\title{
Mental health status of adolescent cancer survivors
}

This article was published in the following Dove Press journal:

Clinical Oncology in Adolescents and Young Adults

18 September 2015

Number of times this article has been viewed

\section{Ann C Mertens Jordan Gilleland Marchak}

Aflac Cancer and Blood Disorder Center, Department of Pediatrics, Emory University School of Medicine, Atlanta, GA, USA
Correspondence: Ann C Mertens Department of Pediatrics, Emory University, 2015 Uppergate Dr,

Atlanta, GA 30322, USA

$\mathrm{Tel}+\mid 404785069$ |

$\mathrm{Fax}+$ I 4047274859

Email amerten@emory.edu
Abstract: Due to the successful treatment of children with cancer, overall 5-year survival rates now exceed $80 \%$. Because of this success in treating childhood cancer, concerns are now focusing on the potential risk of both physical and psychosocial late effects in these cancer survivors. There is limited data available for clinicians and researchers on the mental health of adolescent survivors of childhood cancers. The goal of this review is to provide a concise evaluation of the content and attributes of literature available on this often overlooked, yet vulnerable, population. Overall, studies on psychological outcomes in adolescent survivors of pediatric cancer suggest that the majority are mentally healthy and do not report significant levels of psychological distress. Several factors were recognized as playing an important role in adverse psychosocial outcomes in these adolescent cancer survivors: to include the diagnosis of a tumor in the central nervous system, central nervous system-directed cancer treatment, and physical late effects. To identify the subset of survivors who may benefit from systematic psychological services, systematic psychological screening of all adolescent cancer survivors during followup oncology visits is recommended. Further research into this critical area is needed to help identify other potential risk factors and guide the development of evidence-based support for these vulnerable adolescents.

Keywords: adolescents, psychological, psychosocial, screening recommendations

\section{Introduction}

Due to the successful treatment of children with cancer, overall 5-year survival rates now exceed $80 \% .{ }^{1}$ With this success, however, comes a cost as childhood cancer and its subsequent treatment predispose survivors to a higher risk of certain debilitating diseases and psychosocial problems called late effects of cancer treatment. ${ }^{2}$ Numerous reports and reviews of late effects of chemotherapy and radiation have described sequelae that may present at the end of therapy, shortly following the end of therapy, or years after the completion of therapy. ${ }^{3-5}$ These studies have shown that type and intensity of therapy, as well as the age at therapy, are important factors in determining both overall survival as well as risk for developing late effects. ${ }^{6,7}$ With so many factors contributing to a survivor's late-effect risk profile, individual monitoring for specific physical and psychosocial late effects is complex. ${ }^{8,9}$

To help address these needs within the pediatric cancer survivor population, the Children's Oncology Group (COG) has developed the Long-Term Follow-up Guidelines for Survivor of Childhood, Adolescent, and Young Adult Cancers. ${ }^{10}$ These evidence-based guidelines are risk-based, exposure-related clinical practice guidelines designed for the screening and management of late effects that result from cancer and its treatment. 
The purpose of these guidelines is to provide recommendations for follow-up care that promotes healthy lifestyles and provides ongoing monitoring of health status.

The COG has issued guidelines for the long-term psychological follow-up of all pediatric cancer survivors. ${ }^{11}$ Mental health disorders that are specified in these guidelines as potential psychological late effects include: depression, anxiety, posttraumatic stress, and suicidal ideation. The exposure group for these late effects is any child/adolescent diagnosed with cancer. Personal risk factors identified include female sex and family history of depression, anxiety, or mental illness; social factors include lower household income and lower educational achievement. Treatment/medical risk factors include individuals who receive a hematopoietic cell transplant or experience chronic pain. COG guidelines recommend annual psychological monitoring for all pediatric cancer survivors with periodic reevaluations as clinically indicated. Guidelines also recommend consideration of evaluation of parent/parents for posttraumatic stress symptoms.

Adolescence is a critical period in terms of psychological and social development and is associated with a significant increase in psychological problems in the general population. For survivors of childhood cancer, adolescent development and transition into adulthood can be further complicated by physical and psychosocial late effects of treatment. Adolescents are tackling important developmental challenges during this period including identity development, body-image issues, intimate relationships, separating from parents, and decisions about future goals (ie, education, career). Cognitive and physical vulnerabilities related to the cancer experience and any current late effects of treatment may impact adolescent survivors' ability to face these developmental challenges and increase the risk of developing psychological problems in this period.

There is limited data available for clinicians and researchers on the mental health of adolescent cancer survivors for several reasons. First, because of the limited number of these survivors, research often combines children and adolescents, or adolescents and young adults, into a single research sample. More recent data has largely focused on the mental health status of these survivors once they become adults. And, lastly, due to the diversity in this survivor population (ie, diagnosis, treatment, and age at diagnosis/exposure), studies often generate disparate findings.

The goal of this publication is to provide a concise evaluation of the content and attributes of literature available on this often overlooked, yet vulnerable, population. This is a systematic review of the reported mental health outcomes in adolescents who are survivors of childhood cancer that will serve as a resource for clinicians and researchers working with adolescent cancer survivors. For the purposes of this review, mental health outcomes were defined to include: psychosocial function, emotional concerns and distress, depression, anxiety, and posttraumatic stress. Through this review, we hope to suggest targets for intervention in these adolescents, and identify areas that need further research.

\section{Methods}

A systematic literature search strategy was employed to identify empirical research articles focusing on adolescent survivors of childhood cancer. Prior to beginning the literature search, the authors generated a set of inclusion criteria that studies would need to meet to be included in this review article. The study inclusion criteria for this review included the following: 1) "study content" - the studies must present empirical data (ie, no review articles, policy statements, etc) and the results must be specific to the mental health of adolescent survivors of childhood cancer; and 2) "sample population" - study participants must be 11-20 years at the time of study, have been diagnosed with cancer prior to 18 years of age, and be at least 1 or more years off therapy for pediatric cancer.

We conducted a PubMed search of article titles and abstracts utilizing a combination of four stems containing multiple search terms grouped by theme. These stems were created with the purpose of detecting studies which would meet the review's study inclusion criteria. The first search stem sought to target our adolescent age range and included the terms "adolescent", "adolescence", "pediatric", or "paediatric". The second search stem was implemented to narrow our results to the oncology population and included the terms "oncology", "cancer", "tumor*", or "leukemia". The third search stem was used to identify studies reporting mental health outcomes and included the terms "psychological", "psychosocial", "mental", "emotion*”, "anxiety", "depression", "resilience", "adjustment", "post-trauma*”, or "posttrauma*". Lastly, the fourth search stem was applied in attempt to concentrate results to work done with survivor of cancer and included the terms "survivor*", "off treatment", or "off therapy". The PubMed search results were then limited to articles that were published in peer-reviewed journals, written in English, and published in the past 20 years from the time of this review (ie, from January 1, 1994 to December 31, 2014).

Our PubMed search algorithm with the aforementioned limitations returned a total of $\mathrm{N}=415$ articles. Following 
the systematic PubMed search, the $\mathrm{N}=415$ abstracts were examined to assess if they met the inclusion criteria for this review article. Upon review of the abstract alone, $\mathrm{N}=316$ studies were eliminated due to not meeting inclusion criteria for this review. Examinations of the full text articles were conducted by one author (JGM) for the remaining N=99 studies. Figure 1 outlines the frequencies of the reasons for articles' exclusion from this review. One common reason for exclusion was due to age range of the study sample. Several studies $(\mathrm{N}=60)$ examined adult survivors of child and adolescent cancers, and thus were not appropriate for this review. Many other studies included a wide age range of survivors and did not present results unique to the adolescent age range. In addition, many articles $(\mathrm{N}=25)$ focused only on the mental health of survivors' parents, caregivers, and siblings. Another common reason for exclusion was a lack of specific data on survivors' mental health status; these studies $(\mathrm{N}=118)$ did not report mental health outcomes but were instead dedicated to measure development, general health-related quality of life (HRQOL), or medical and/or neurocognitive outcomes only. Several articles did not present any empirical data, and thus these review articles $(\mathrm{N}=60)$ and other papers $(\mathrm{N}=43$; case studies, clinical guidelines, etc) were excluded from the current review. Ultimately, $\mathrm{N}=17$ articles from the PubMed search were found to meet criteria for inclusion for review in the current paper (Table 1). A synopsis of each article is detailed later.

\section{Results \\ Psychosocial functioning}

Psychological and social functioning can often be captured as a part of assessments of overall HRQOL. A population-based study conducted in Norway provides a comprehensive description of quality of life and mental health as reported by

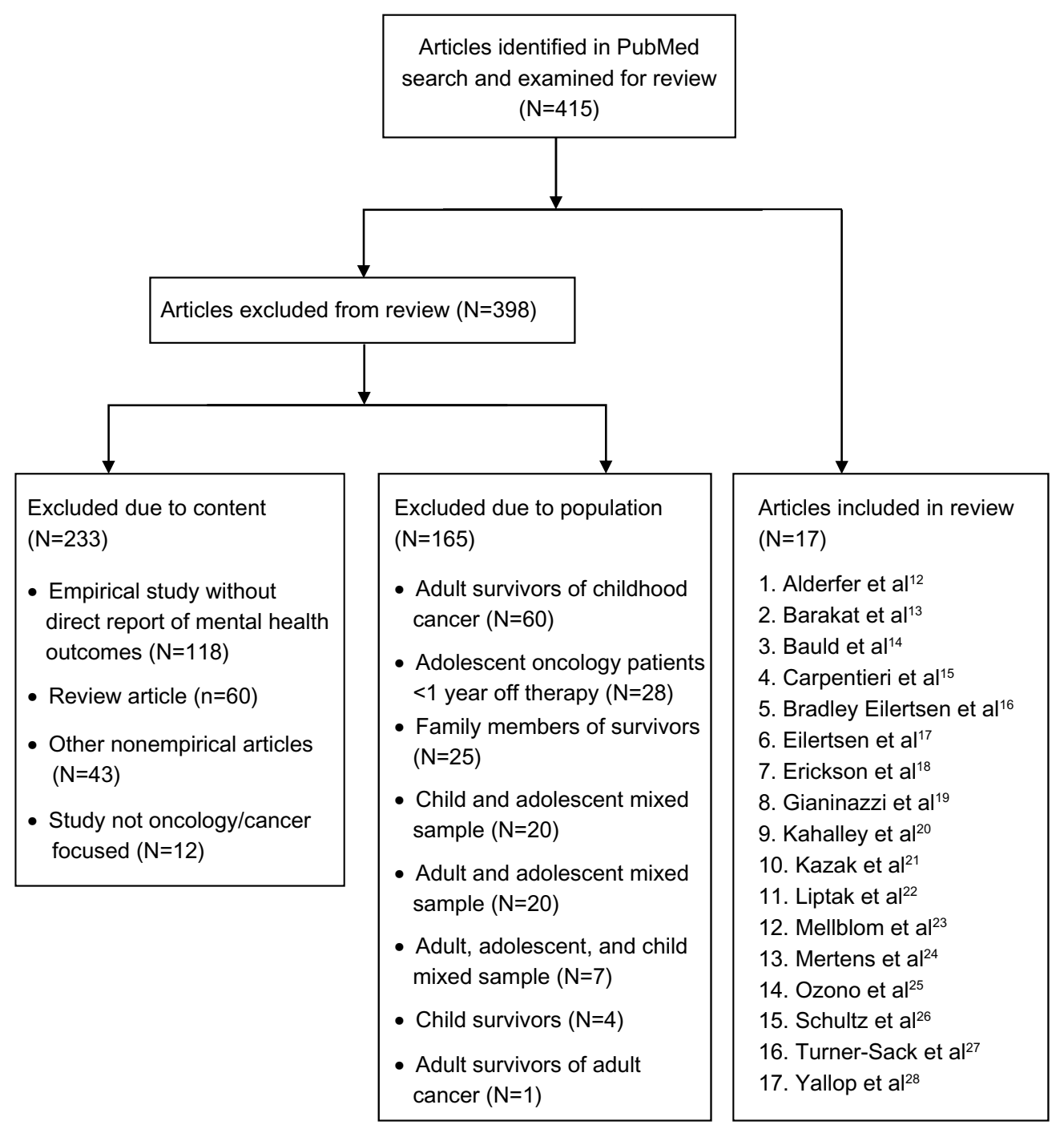

Figure I Article exclusion criteria. 


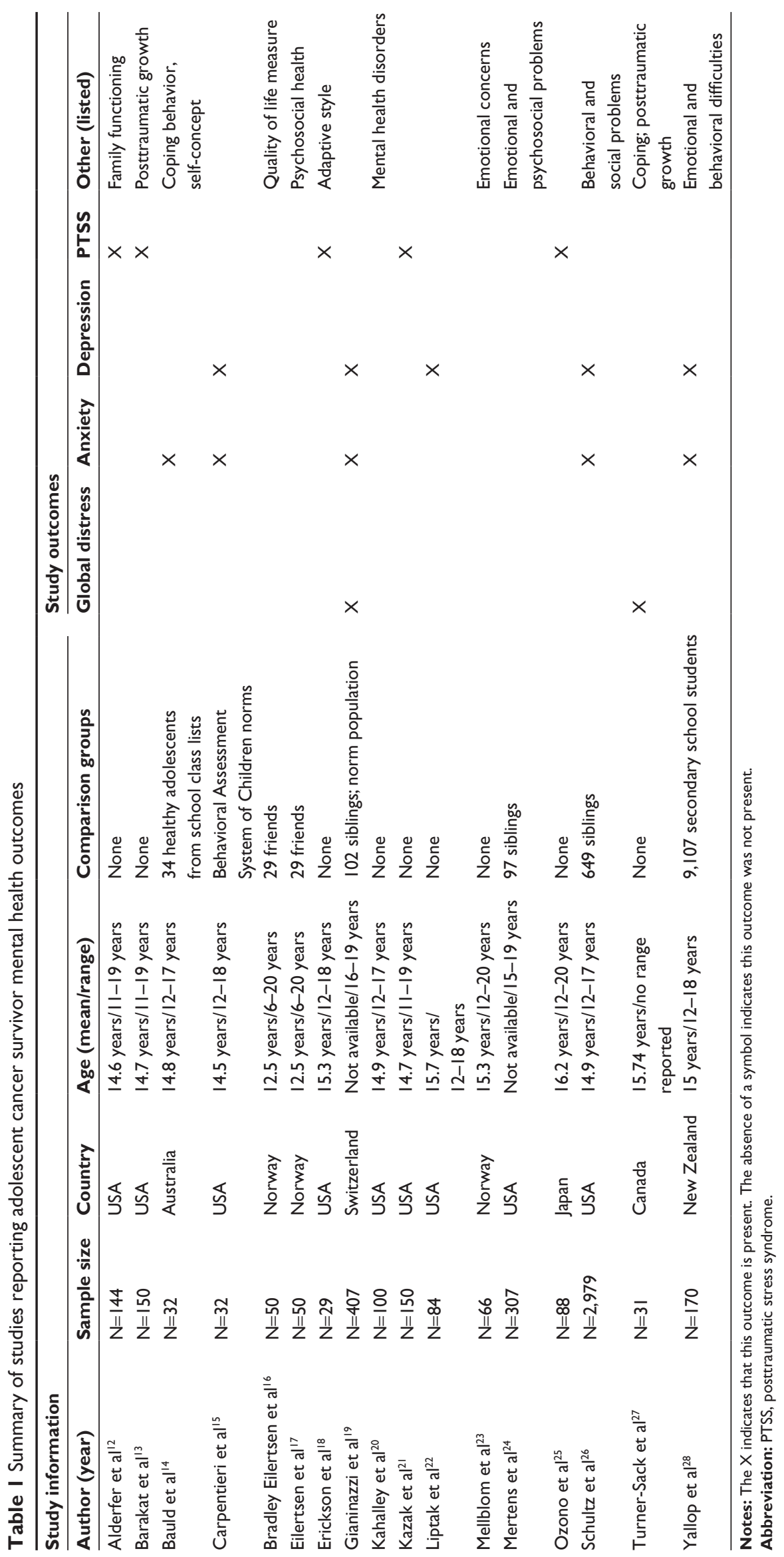


adolescent survivors and their parents. ${ }^{16}$ Mailed surveys were distributed to adolescent survivors who were at least 3 years off therapy; a friend invited by the survivor was utilized as a comparison population. In the adolescent self-report on the Inventory of Life Quality on Children and Adolescents measure, both the parent report and the survivor's report showed no significant difference in overall quality of life scores as compared to healthy friend controls. Parents reported significantly lower mental health scores among survivors overall and within specific diagnoses of leukemia and brain tumors when compared to controls. Survivors reported similar mental health scores with all cancer diagnoses combined, except for brain tumor survivors who reported poorer mental health when compared to controls.

This Norwegian population study also looked at specific domains of psychosocial health as assessed by the Strengths and Difficulties Questionnaire (SDQ), a psychosocial behavioral screening tool. ${ }^{17}$ On the SDQ total psychosocial difficulties score, parents reported that adolescent survivors had significantly poorer scores when compared to healthy controls, while the report of adolescents showed no differences between survivors and healthy controls. On the emotional symptom scale, adolescent survivors reported poorer outcomes than controls. Adjusting for sex and parental marital status did not change the results on the adolescent self-report. In addition, adolescent survivors with parent-reported late effects had higher SDQ mean scores in total difficulties, emotional symptoms, and peer problem scales than controls. Internal comparison showed that these adolescent survivors with late effects also reported higher scores on the peer problem subscale than those without late effects.

In an ancillary study through the Childhood Cancer Survivor Study, adolescents between the ages of 15 and 19 years were asked to self-report on the Child Health and Illness Profile - Adolescent Edition survey, designed to assess adolescent HRQOL, including emotional and psychosocial disorders. ${ }^{24}$ Overall, as compared to siblings, survivors reported similar rates of emotional discomfort; in addition, Wilms' tumor survivors reported less emotional discomfort than sibling controls. When comparing survivors, females reported poorer functioning than male survivors. Overall, survivors also reported poorer scores for psychosocial disorders when compared to siblings. This was particularly significant among survivors diagnosed with leukemia, central nervous system (CNS) tumors, and neuroblastoma.

The Childhood Cancer Survivor Study also utilized the parent-reported Behavior Problem Index to assess the behavioral and social outcomes of adolescents who were at least 5 years off therapy and were between 12 and 17 years of age. ${ }^{26}$ Domains included: depression/anxiety, headstrong, attention deficit, peer conflict/social withdrawal, antisocial, and social competence. Overall, most survivors were perceived by parents to be functioning well; however, survivors scored poorer in all measured domains than seen in a comparable sibling group. Scores in the depression/anxiety, attention deficit, and antisocial domains were significantly elevated in adolescents treated for leukemia and CNS tumors when compared to siblings. Similarly, survivors reporting a disfigurement also had elevated scores in the depression/ anxiety, attention deficit, and antisocial domains. When compared to survivors who did not have CNS treatment, those who were treated with either cranial radiation or both intrathecal methotrexate and cranial radiation were at increased risk of depression/anxiety, attention deficit, antisocial behaviors, and diminished social competence.

\section{Emotional concerns and distress}

Several studies utilized only self-report within adolescent cancer survivor populations to assess aspects of mental health. An internet survey distributed to childhood cancer survivors between 12 and 18 years of age was developed to describe the self-reported psychosocial well-being of adolescent cancer survivors using the World Health Organization-Five Wellbeing Index, the Reynolds Adolescent Depression Sale, the SDQ, and the Multidimensional Anxiety Scale for Children. ${ }^{28}$ Results showed the vast majority of adolescent cancer survivors reported scores within the normal range for anxiety (93\%), depression (94\%), well-being (89\%), and emotional/behavioral difficulties (82\%). No statistically significant differences were found between either anxiety or depressive symptoms and any demographic (sex, ethnicity, age at study) or cancer characteristics (diagnosis, age at diagnosis, time since diagnosis, cancer treatment). When compared with secondary school students of similar age, adolescent cancer survivors were less likely to report abnormal psychosocial well-being (11\% vs $21 \%)$ or depressive symptoms ( $6 \%$ vs $11 \%$ ).

In an effort to better contextualize the severity of distress in survivors, a Swiss study mailed questionnaires to children diagnosed with cancer before the age of 16, with comparisons made to siblings, a normative population, and to psychotherapy patients within the same age group. ${ }^{19}$ Using the Brief Symptoms Inventory (BSI-18), this study showed that overall, the majority of survivors had little or no significant psychological distress, with $13 \%$ of survivors and $11 \%$ of siblings reporting clinical distress. However, 
survivors had higher scores in the global severity index and on the somatization, depression, and anxiety subscales as compared to the normative population. More females than males reported symptoms of psychological distress. When comparing actual scores ( $t \geq 57$ in the respective scale), distressed survivors scored significantly higher than distressed siblings on the somatization subscale and the global severity index; distressed survivors also had higher scores than distressed psychotherapy patients on somatization and anxiety subscales. Multivariable models showed that determinants for psychological distress in survivors were female sex, self-reported somatic and psychological late effects, and low perceived parent support.

Turner-Sack et al conducted an examination of the relationships between psychological distress, coping, and posttraumatic growth among adolescent survivors. ${ }^{27}$ Their study found that the overall mean level of distress reported by survivors on the BSI-18 was within the average range. No association was found between psychological distress on the BSI-18 and posttraumatic growth as measured by the Posttraumatic Growth Inventory. Higher levels of psychological distress were related to older age at diagnosis and use of avoidant coping strategies.

In a study investigating the psychological needs of adolescent survivors of acute lymphoblastic leukemia (ALL) and brain tumors, adolescent survivors were evaluated using the Diagnostic Interview for Children and Adolescents IV. ${ }^{20}$ This assessment indicated that $82 \%$ of these adolescent survivors exhibited psychological concerns including significant problems with cognitive (47\%), behavioral (76\%), and/or emotional (19\%) functioning on psychological assessments. Exposure to cranial radiation was associated with more cognitive concerns; lower income was associated with cognitive concerns in ALL survivors. In addition, this study found that many of these adolescent survivors experience psychological difficulties that are not adequately managed by current services.

Importantly, it is also critical to ascertain whether adolescent cancer survivors are able to express their emotional concerns during their visits to their oncology providers. One qualitative study used video recordings to investigate what concerns adolescent survivors of either leukemia or lymphoma communicated to their treating oncologist. ${ }^{23}$ Of the 66 children who participated, 50\% expressed emotional concerns, of which $29 \%$ were psychological concerns. These cues/concerns were most often elicited by the oncologist participating in the consultation, confirming that these concerns should be actively explored in follow-up consultations. Albeit, with a limited sample, findings suggest that adolescents were more likely to express concerns when they were alone with the oncologists, rather than when a parent was also present during the consultation.

\section{Anxiety and depression}

In a small study of adolescent survivors of pediatric cancer, most with a diagnosis of ALL, psychosocial status was evaluated by assessing anxiety levels using the State-Trait Anxiety Inventory, coping mechanisms using the Adolescent Coping Scale, and self-concept using the Self-Description Questionnaire II. ${ }^{14}$ When compared to healthy controls, adolescent survivors demonstrated overall higher scores for anxiety proneness (state anxiety), but not for current levels of tension and apprehension (trait anxiety). No differences were seen in overall coping levels between survivors and controls; however, significant differences were noted for "wishful thinking" and "ignore the problem" strategies, suggesting a tendency among survivors to employ more nonproductive/ avoidance strategies than healthy controls.

Self-, parent-, and teacher-report of psychological functioning as measured by the Behavioral Assessment System for Children was obtained within a sample of adolescent survivors of brain tumors. ${ }^{15}$ When compared to general population norms from the Behavioral Assessment System for Children, no elevations in the proportion of patients with "at-risk" scores for anxiety or depression were found among survivor, parent, or teacher reports.

Symptoms of depression, anxiety, and problematic selfconcept were assessed among adolescent survivors using the Beck Youth Inventory-II as well as through semistructured clinical interviews. ${ }^{22}$ Clinically significant problems were reported by survivors related to anxiety (7\%), depression $(6 \%)$, and self-concept (10\%). Overall, $17 \%$ of adolescents in the sample reported at least one clinical elevation in anxiety, depression, or self-concept. Of those adolescents who reported at least one clinically elevated subscale $(\mathrm{N}=14)$, only $57 \%(\mathrm{~N}=8)$ were identified by clinicians as experiencing significant distress.

\section{Posttraumatic stress}

Within a sample of adolescent cancer survivors, 8.3\% met criteria for posttraumatic stress disorder (PTSD) based on the results of the Structured Clinical Interview for the $D S M-I V .{ }^{12}$ Half of adolescent survivors (47\%) and over one-third of parents (35\% mothers, 38\% fathers) reported significantly poor general family functioning as measured by the Family Assessment Device. Adolescents with PTSD were more than 
five times as likely to emerge from a poorly functioning family, and $75 \%$ of the survivors with PTSD came from families with categorically poor family functioning.

Analyses of self-reported data demonstrated a strong association between posttraumatic stress syndrome (PTSS) endorsed on the Impact of Events Scale-Revised and posttraumatic growth among adolescent survivors. ${ }^{13}$ Over $80 \%(84.7 \%)$ of adolescents reported at least one positive consequence related to their cancer experience on the Perceptions of Changes in Self scale of the Impact of Traumatic Stressors Interview Schedule, while almost one-third (32\%) of adolescents reported four or more positive outcomes on the Perceptions of Changes of Self scale. Adolescent posttraumatic growth was also associated with older age at diagnosis and perceived treatment intensity and life threat.

Erickson et al sought to evaluate differences in PTSS reported by adolescent survivors based on social-emotional adaptive style. ${ }^{18}$ Social-emotional adaptive style was assessed in this study via self-report using the Weinberger Adjustment Inventory, while PTSS was measured using results from a semistructured interview, the Clinician-Administered PTSD Scale for Children and Adolescents. Based on their interviews, $13.8 \%$ of the sample met criteria for PTSD. Adolescent survivors who reported an adaptive style characterized by decreased awareness of distress and increased self-restraint endorsed lower levels of PTSS on the Clinician-Administered PTSD Scale for Children and Adolescents. In contrast, no differences were found between adolescents with different adaptive styles on scores of PTSS measured via self-report on the Child Posttraumatic Stress Reaction Index Revision 2.

An investigation of PTSS and PTSD among adolescent cancer survivors demonstrated that $17.6 \%$ of survivors reported moderate to severe levels of PTSS on the Posttraumatic Stress Disorder Reaction Index. ${ }^{21}$ In addition, 4.7\% of adolescent survivors currently met the full criteria for PTSD as evaluated by the Structured Clinical Interview for the Diagnostic and Statistical Manual of Mental Disorders, while $8.0 \%$ of survivors met criteria for PTSD at some point in time since their cancer diagnosis. Survivors frequently reported symptoms of reexperiencing $(73.3 \%)$, followed by symptoms of arousal (41.3\%) and avoidance (16.1\%).

An investigation of PTSS among Japanese adolescent cancer survivors found that $10.9 \%$ of their sample reported severe levels of PTSS as measured by the Impact of Events Scale-Revised. ${ }^{25}$ A significant correlation between adolescents self-report of PTSS and their mothers' self-reported PTSS was observed. Results of multivariate analyses indicated that higher trait anxiety and experiencing late effects of treatment or other disease were significant predictors of adolescent PTSS.

\section{Discussion}

Through this systematic review of reported mental health outcomes in adolescent cancer survivors, we identified 17 articles that met our literature search criteria. Studies on psychological outcomes in adult survivors of pediatric cancer suggest that the majority are mentally healthy and do not report psychological distress. ${ }^{4,29}$ In this review, we found the same for adolescent survivors, with approximately 10\%-20\% of survivors consistently reporting various psychological difficulties. ${ }^{18-21,23,25,28}$ More specifically, global problems with distress and general emotional functioning were reported by $13 \%-29 \%$ of adolescent survivors. ${ }^{19,20,22,23,28}$ Rates of clinical anxiety (7\%) and depression (6\%) were consistent between the two investigations reporting specific measures of these constructs. ${ }^{22,28}$ Finally, the diagnostic rates of PTSD in the adolescent samples included in this review ranged from $8.0 \%$ to $13.8 \% ;^{12,18,21}$ however, rates of self-reported symptoms of posttraumatic stress were higher. ${ }^{21,25}$ Symptoms of depression or anxious avoidance may lead to nonadherence with longterm follow-up care, while somatic symptoms associated with PTSD, anxiety, and depression may lead to increased or unnecessary health care utilization. ${ }^{21}$ Given the prevalence and impact of these negative mental health outcomes in this population, proper assessment and identification of psychological symptoms among adolescent survivors is a crucial component of psychosocial survivor care.

In terms of overall mental health status, previous literature has suggested that pediatric cancer patients can experience both positive and negative psychosocial outcomes after completion of treatment, and that these experiences are not mutually exclusive. ${ }^{9}$ These dialectal findings may help explain some of the contrasts in adolescent survivor mental health outcomes seen between the $\mathrm{N}=8$ investigations in this review that included adolescent report and a comparison group. Three of these studies described mixed outcomes in which survivors reported worse functioning in some psychosocial domains and no differences in other domains as compared to controls. ${ }^{14,17,24}$ Two other studies found no differences in adolescent report of mental health outcomes between survivors and healthy controls. ${ }^{15,16}$ Lastly, one study showed adolescent survivors reporting worse distress as compared to norms, ${ }^{19}$ while another study found adolescent survivors' report of psychological well-being to be better than peers. ${ }^{28}$

The adult childhood cancer survivor literature indicates that certain groups of childhood cancer survivors are at high risk for psychological distress and poor HRQOL, especially 
in physical domains. Risk factors for psychological distress and poor HRQOL among adult survivors of pediatric cancer are female sex, annual household income less than $\$ 20,000$, lack of health insurance, presence of a major medical condition, and treatment with cranial radiation and/or surgery. ${ }^{4}$ Similarly, many demographic and medical factors were recognized in these pediatric studies as playing an important role in psychosocial outcomes in adolescent cancer survivors. Several of the studies included in this review suggested that diagnoses of leukemia or CNS tumors were significant contributing factors to poorer psychosocial outcomes and psychological difficulties in adolescent cancer survivors; ${ }^{16,20,24,26}$ however, it is important to note that one study found no differences between brain tumor survivors and population norms on measures of anxiety or depression. ${ }^{15}$ Older age at diagnosis was associated with greater levels of global distress, ${ }^{27}$ as well as increased reported posttraumatic growth. ${ }^{13}$ Female sex was related to increased distress and emotional discomfort. ${ }^{19,24}$ Self-reported late effects of treatment were linked to increased global distress, emotional difficulties, peer problems, and PTSS among adolescent survivors. ${ }^{17,19,25}$ Finally, the literature indicates that that there are significant relationships between posttraumatic stress in adolescent survivors of childhood cancer and many other psychosocial factors including their family functioning, adaptive style, trait anxiety, and posttraumatic growth. ${ }^{12,13,18,25}$

The results of review also demonstrate that proxy reports by parents were more likely to describe poorer psychological functioning among adolescent cancer survivors than that described by parents of comparison-group adolescents. ${ }^{16,17,26}$ Of interest, notable differences were seen within these same studies, showing that adolescent survivor self-report scores of psychological symptoms were comparable to scores seen in the adolescent comparison groups. These findings likely suggest a different perspective between parental and adolescent's experience and perception of their health, both of which are important when attempting to further understand mental health in this vulnerable population. These differences in perspectives may be partially attributable to increased PTSS typically observed among parents of survivors. ${ }^{21,25}$ Given that survivors typically report worse psychosocial outcomes than their siblings, ${ }^{19,24,30}$ these differences between adolescent and parent report may also be a product of parental comparison between survivors and their siblings.

\section{Limitations to study findings}

There are four major factors that contributed to the diversity of study results available for consideration in this review. First, a variety of survey instruments were used to determine mental health outcomes in these adolescent cancer survivors, contributing to the difficulty in coming to a definitive consensus. Second, differences in findings between studies may be due to control groups selected for comparison purposes (ie, school vs friend vs normative population controls) or no comparison group. Also, many studies struggle with a diverse/heterogeneous group of cancer survivors (ie, differences in age at diagnosis, diagnosis and intensity of cancer treatment) and small sample size. Lastly, selection bias needs to be taken into consideration as some studies reported in this review required the teen to answer the survey, which may have excluded those with difficulties responding to a survey because of vision and/or cognitive problems, thereby under representing those who may have reported poorer psychological functioning.

\section{Implications/future direction}

Because of the diversity within the studies, and the limited number of studies available to review for this topic, it is difficult to draw many overarching conclusions related to the mental health status of adolescent survivors of childhood cancer. Further research into this critical area could benefit from survey tools, such as the Patient Reported Outcomes Measurement Information System, a system of measures developed to collect patient-reported health status for physical, mental, and social well-being. ${ }^{31}$ The consistent use of consistent measures across the continuum of adolescent cancer survivors would provide a better opportunity to understand how various cancers and their treatments might affect survivors, identify adolescent survivors at risk, and ultimately improve mental health outcomes.

\section{Conclusion}

Findings from this systematic review reinforce the COG guidelines which recommend annual psychological monitoring for all pediatric cancer survivors with periodic reevaluations as clinically indicated. Although most survivors report good psychological health, there is a subset of survivors who are reporting significant difficulties. Systematic psychological screening will help health care providers more effectively identify these patients. Psychological screening during oncology visits followed by appropriate linking to psychosocial services is crucial to supporting vulnerable adolescents in this phase of their development into adulthood. Oncology providers should consider outreach and collaboration with behavioral health professionals to implement evidence-based screening and facilitate access to mental health services in 
order to best address the needs of childhood cancer survivors in terms of their psychological well-being.

\section{Disclosure}

The authors report no conflicts of interest in this work.

\section{References}

1. SEER Cancer Statistics Review, 1975-2011 [webpage on the Internet]. Bethesda: National Cancer Institute; 2014. Available from: http://seer. cancer.gov/csr/1975_2011/. Accessed April 29, 2015.

2. Oeffinger KC, Hudson MM. Long-term complications following childhood and adolescent cancer: foundations for providing risk-based health care for survivors. CA Cancer J Clin. 2004;54(4):208-236.

3. Hudson MM, Mulrooney DA, Bowers DC, et al. High-risk populations identified in Childhood Cancer Survivor Study Investigations: implications for risk-based surveillance. J Clin Oncol. 2009;27(14): 2405-2414.

4. Zeltzer LK, Recklitis C, Buchbinder D, et al. Psychological status in childhood cancer survivors: a report from the Childhood Cancer Survivor Study. J Clin Oncol. 2009;27(14):2396-2404.

5. Ness KK, Hudson MM, Ginsberg JP, et al. Physical performance limitations in the Childhood Cancer Survivor Study Cohort. J Clin Oncol. 2009;27(14):2382-2389.

6. Pogany L, Barr RD, Shaw A, Speechley KN, Barrera M, Maunsell E. Health status in survivors of cancer in childhood and adolescence. Qual Life Res. 2006;15(1):143-157.

7. Dickerman JD. The late effects of childhood cancer therapy. Pediatrics. 2007;119(3):554-568.

8. Oeffinger KC, Hudson MM, Landier W. Survivorship: childhood cancer survivors. Prim Care. 2009;36(4):743-780.

9. Wakefield CE, McLoone J, Goodenough B, Lenthen K, Cairns DR, Cohn RJ. The psychosocial impact of completing childhood cancer treatment: a systematic review of the literature. J Pediatr Psychol. 2010;35(3):262-274.

10. Landier W, Bhatia S, Eshelman DA, et al. Development of risk-based guidelines for pediatric cancer survivors: the Children's Oncology Group Long-Term Follow-Up Guidelines from the Children's Oncology Group Late Effects Committee and Nursing Discipline. J Clin Oncol. 2004;22(24):4979-4990.

11. The Children's Oncology Group Long-Term Follow-Up Guidelines for Survivors of Childhood, Adolescent and Young Adult Cancers [webpage on the Internet]. Monrovia: Children's Oncology Group. Available from: http://www.childrensoncologygroup.org/index.php/survivorshipguidelines. Accessed April 29, 2015.

12. Alderfer MA, Navsaria N, Kazak AE. Family functioning and posttraumatic stress disorder in adolescent survivors of childhood cancer. J Fam Psychol. 2009;23(5):717-725.

13. Barakat LP, Alderfer MA, Kazak AE. Posttraumatic growth in adolescent survivors of cancer and their mothers and fathers. J Pediatr Psychol. 2006;31(4):413-419.

14. Bauld C, Anderson V, Arnold J. Psychosocial aspects of adolescent cancer survival. J Paediatr Child Health. 1998;34(2):120-126.
15. Carpentieri SC, Meyer EA, Delaney BL, et al. Psychosocial and behavioral functioning among pediatric brain tumor survivors. J Neurooncol. 2003;63(3):279-287.

16. Bradley Eilertsen ME, Jozefiak T, Rannestad T, Indredavik MS, Vik T. Quality of life in children and adolescents surviving cancer. Eur J Oncol Nurs. 2012;16(2):185-193.

17. Eilertsen ME, Rannestad T, Indredavik MS, Vik T. Psychosocial health in children and adolescents surviving cancer. Scand J Caring Sci. 2011;25(4):725-734.

18. Erickson SJ, Gerstle M, Montague EQ. Repressive adaptive style and self-reported psychological functioning in adolescent cancer survivors. Child Psychiatry Hum Dev. 2008;39(3):247-260.

19. Gianinazzi ME, Rueegg CS, Wengenroth L, et al. Adolescent survivors of childhood cancer: are they vulnerable for psychological distress? Psychooncology. 2013;22(9):2051-2058.

20. Kahalley LS, Wilson SJ, Tyc VL, et al. Are the psychological needs of adolescent survivors of pediatric cancer adequately identified and treated? Psychooncology. 2013;22(2):447-458.

21. Kazak AE, Alderfer M, Rourke MT, Simms S, Streisand R, Grossman JR. Posttraumatic stress disorder (PTSD) and posttraumatic stress symptoms (PTSS) in families of adolescent childhood cancer survivors. J Pediatr Psychol. 2004;29(3):211-219.

22. Liptak C, Manley P, Recklitis CJ. The feasibility of psychosocial screening for adolescent and young adult brain tumor survivors: the value of self-report. J Cancer Surviv. 2012;6(4):379-387.

23. Mellblom AV, Finset A, Korsvold L, Loge JH, Ruud E, Lie HC. Emotional concerns in follow-up consultations between paediatric oncologists and adolescent survivors: a video-based observational study. Psychooncology. 2014;23(12):1365-1372.

24. Mertens AC, Brand S, Ness KK, et al. Health and well-being in adolescent survivors of early childhood cancer: a report from the Childhood Cancer Survivor Study. Psychooncology. 2014;23(3):266-275.

25. Ozono S, Saeki T, Mantani T, et al. Psychological distress related to patterns of family functioning among Japanese childhood cancer survivors and their parents. Psychooncology. 2010;19(5):545-552.

26. Schultz KA, Ness KK, Whitton J, et al. Behavioral and social outcomes in adolescent survivors of childhood cancer: a report from the childhood cancer survivor study. J Clin Oncol. 2007;25(24):3649-3656.

27. Turner-Sack AM, Menna R, Setchell SR, Maan C, Cataudella D. Posttraumatic growth, coping strategies, and psychological distress in adolescent survivors of cancer. J Pediatr Oncol Nurs. 2012;29(2):70-79.

28. Yallop K, McDowell H, Koziol-McLain J, Reed PW. Self-reported psychosocial wellbeing of adolescent childhood cancer survivors. Eur J Oncol Nurs. 2013;17(6):711-719.

29. Zebrack BJ, Zeltzer LK, Whitton J, et al. Psychological outcomes in long-term survivors of childhood leukemia, Hodgkin's disease and nonHodgkin's lymphoma: a report from the Childhood Cancer Survivor Study. Pediatrics. 2002;110:42-52.

30. Zeltzer LK, Lu Q, Leisenring W, et al. Psychosocial outcomes and health-related quality of life in adult childhood cancer survivors: a report from the childhood cancer survivor study. Cancer Epidemiol Biomarkers Prev. 2008;17(2):435-446.

31. PROMIS ${ }^{\circledR}$ Overview [webpage on the Internet]. Chapel Hill: PROMIS Network Center. Available from: http://www.nihpromis.org/about/ overview. Accessed April 29, 2015.
Clinical Oncology in Adolescents and Young Adults

\section{Publish your work in this journal}

Clinical Oncology in Adolescents and Young Adults is an international, peer-reviewed, open access journal publishing original research, reports, editorials, reviews and commentaries on all aspects of epidemiology, diagnosis and treatment of cancers in adolescents and young adults. The manuscript management system is completely

\section{Dovepress}

online and includes a very quick and fair peer-review system. Visit http://www.dovepress.com/testimonials.php to read real quotes from published authors. 